\title{
Do distant or colocated audiences affect user activity in VR?
}

\author{
Romain Terrier ${ }^{1,2}$, Nicolas Martin ${ }^{1}$, Jeremy Lacoche ${ }^{3}$, Valerie Gouranton ${ }^{2}$, and \\ Bruno Arnaldi ${ }^{2}$ \\ 1 IRT b-com, Rennes, France \\ romain.terrier@b-com.com \\ 2 Univ Rennes, INSA Rennes, Inria, CNRS, IRISA, Rennes, France \\ 3 Orange Labs, Rennes, France
}

\begin{abstract}
We explore the impact of distant or colocated real audiences on social inhibition through a user study in virtual reality (VR). The study investigates, in an application, the differences among two multiuser configurations (i.e., the local and distant conditions) and one control condition where the user is alone (i.e., the alone condition). In the local condition, a single user and a real audience share the same real room. Conversely, in the distant condition, the user and the audience are separated into two different real rooms. The user performed a categorization of numbers task in VR, for which the users' performance results (i.e., type and answering time) are extracted as subjective feelings and perceptions (i.e., perceptions of others, stress, cognitive workload, presence). The differences between the local and distant configurations are explored. Furthermore, we investigate any gender biases in the objective and subjective results. During the local and distant conditions, the presence of a real audience affects the user's performance due to social inhibition. The users are even more influenced when the audience does not share the same room, despite the audience being less directly perceived in this condition.
\end{abstract}

Keywords: Virtual Reality · Social influence · Audience

\section{Introduction}

In virtual reality (VR), the new trend is toward multi-users [43]. People are able to share, in real time, the same virtual rnvironment (VE) and their experience causing social mechanisms [6] (e.g., social anxiety, social inhibition, empathy, group effects, leadership). Moreover, social VR applications allow people to share the same VE in different ways. On the one hand, they can be colocated when each user is in the same location. On the other hand, when each user is in a different, remote location, they are distant. This differentiation can affect the user's possible degree of social inhibition; indeed, neither the effects nor the appearance of social inhibition are currently well understood in VR. Therefore, one question remains: does our behavior change in VR depending on whether our 
audience is physically present or remotely located? Using previous experimental studies in psychology, we designed an experiment in which one user must perform a new and unknown task in front of distant or colocated others. In each condition, this audience shares the same VE as the user (see Fig. 1). The goal of this study is to analyze the differences in social inhibition depending on the location of the audience. This is an extended version of the paper invited to SI on CGI 2019 [41].
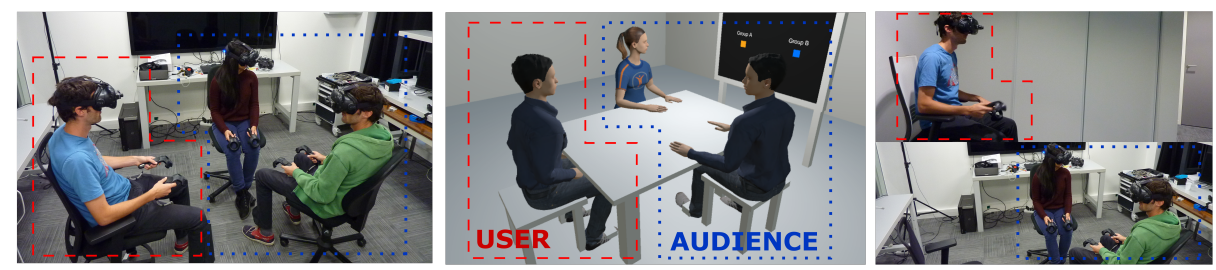

Fig. 1: User: red dashes. Audience: blue dots. A single shared virtual environment (VE) (middle) for two different conditions: distant (right) and colocated (left).

\section{Related Work}

\subsection{Social influence and audience effects in the real world}

In the real world, everyday tasks are often done in the presence of other people or in cooperation with them. The perception an individual has of these other people affects his or her actions and behaviors [14] by imitation effects [11] or by a priming effect [37].

First, the mere presence of other people (an audience) is enough to influence individual behaviors [47] and to cause social facilitation [13] or social inhibition. The audience acts like an amplifier: it increases the dominant response, which is task complexity-dependent, of the user. Consequently, the realization of an easy or well-known task will be facilitated by the mere presence of an audience, a concept known as facilitation [20]. In contrast, the realization of a complex or new task requiring a learning phase will be impaired by the presence of an audience [46], an effect known as social inhibition.

However, social inhibition or facilitation are not only generated by the mere presence of an audience [21]. How the audience is perceived or interpreted also affects the user, whose performance varies depending on the audience's status (i.e., as an evaluative or non-evaluative audience) [24]. Indeed, the user performs worse when facing an evaluative audience [26] than when alone because of the "evaluation apprehension" effect. A user may perceive an experimenter as an expert, and thus as an evaluative auditor, even if the experimenter is just an observer [39]. Moreover, knowing the identity of the audience (e.g., an audience composed of the user's friends or colleagues) reduces users' accuracy and answering time [45]. Social norm effects can also influence other behaviors: in a 
risky situation and in the presence of an audience users tend to diminish their "risk-taking" actions (e.g., in poker gambling) [30].

Thus, many studies have been published on the social effects of an audience on a user, including those generated by the mere presence of the audience, the user's perception of the audience, and the predictability of the audience, along with other effects relating to evaluation apprehension, social norms, and distraction. All such studies refer to social facilitation or inhibition. Moreover, the social influence of a virtual audience on a user could be different when compared to the influence of a real audience due to the particularities of VR.

\subsection{VR: a medium for social interaction studies}

Multiple users can share the same VE in real time [15] and can be physically situated either in the same room (i.e., colocated) or at a remote location (i.e., distant). In shared virtual environments (SVEs), each user is represented by an avatar. Avatars are distinguished from embodied agents "which are models driven by computer algorithms" [5].

By sharing the same VE, the use of avatars supports social interactions through non-verbal communication (e.g., head movements [3], gaze tracking [18]) and copresence (i.e., "being there together" [32]). For this reason, researchers have used SVEs to study users' behaviors in VR (e.g., paranoia, phobia, stress, anxiety) [31]. For example, the VE is able to induce anxiety in people suffering from social phobia [22]. In such contexts, the user is able to calm down by visualizing their doppelganger (i.e., a virtual copy of themselves ) [4] speaking in front of others in the VE [2].

The aim of these studies is to reproduce real-life situations in VR (e.g., sport training [1]) or to analyze real interaction [33] and communication in VR. Other studies have replicated psychological results obtained in real environments in VEs (e.g., the Milgram experiment [35]).

In this way, VR is a valid tool for mirroring real social processes in the VE [10] and for studying social effects.

\subsection{Social inhibition in VR}

Since SVEs induce co-presence, they have been used to support studies on users' behavior. Participants are either physically in the same room (i.e., colocated) or in a remote location (i.e., distant). Several studies $[16,44]$ on social influence focus on analyzing social inhibition in VR. Social inhibition occurs when one performs a new or unknown task in front of an audience, resulting in a decrease in performance (e.g., slower performance and poorer qualitative and quantitative results) [8].

Many VR studies specialize in researching the type of audience (e.g., real versus virtual in $2 \mathrm{D}$ or $3 \mathrm{D}$, close versus far). The first studies on social inhibition of return (e.g., increase in answering time in front of a co-actor) found that the presence of an agent in the VE caused a longer response time (e.g., 20ms) [44]. 
A previous study found that only the presence of a real co-actor caused this effect compared to a 2D-agent displayed on TV [34]. Moreover, a user study [25] focused on the difference of impact among three types of audience condition: alone, with avatars, and with agents. The study is based on the previous works of Blascovich et al. [7], who found that users provided fewer correct answers when executing the unlearned task in the presence of an audience. Blascovich et al. also discovered a correlation between physiological patterns and social inhibition: when a participant was performing the task in the presence of others, measurements of the participant's cardiovascular reactivity could be associated with a threat pattern of physiological reactivity. In the Hoyt et al. study [25], social inhibition occurred in VR but not social facilitation, and only in the presence of avatars. Indeed, if agents are judged and perceived as non-evaluative, the emergence of such inhibition can be prevented [13]. Moreover, virtual agents exert less social influence than avatars on user feelings and behaviors [40], resulting in non-existent or low social inhibition. In the Hoyt et al. study [25], lower performance (e.g., a lower number of correct answers during a novel task) was caused by social inhibition. However, given that the assistant stayed in the room even if the condition was without an audience, their presence could have had an impact on results.

There is still uncertainty regarding the effect of the audience type on users. Some results have shown social inhibition in the presence of an agent, while others have indicated social inhibition only in the presence of an avatar in a VE. One limitation of these previous studies is that the effect of co-presence is often not measured, reducing data obtained on the possible effects of the perception of others in a VE. Given that an audience in a VE can induce evaluation apprehension and self-evaluation effects on users, it can influence users' perceived stress [29] and workload [12] during the task accomplishment.

In summary, studies have demonstrated the impact of a virtual audience on a user depending on its type (i.e., avatar, agent) and the user's perception of it (i.e., as evaluative or non-evaluative). However, there is a lack of studies that consider the location of the audience, although the issue of physical distance has always been confronted [28]. Today, applications can be shared and users can be located remotely (i.e., distant) or in the same room (i.e., colocated). Therefore, our study proposes to address this topic through analyzing how different audience locations affect the impact of social inhibition.

\section{Our social inhibition experiment}

The aim of this experiment is to evaluate the impact of an audience's location (i.e., colocated, distant) on users in a VE. The audience takes the form of two examiners in the VE to establish the social inhibition of the participant. This paper does not focus on social facilitation because past studies have only found results on inhibition [25]. The study is a between-subjects design with one independent variable: the presence of examiners. The participant performs the task according to three conditions: (1) alone, with no audience present (ALONE); 
(2) with the examiners present as a colocated audience, sharing the VE and the same real room as the participant (IN), (3) with the examiners as a distant audience, sharing the VE but not the same real room (OUT) (see Fig. 2). In conditions 2 and 3, the examiners and the user wear a head-mounted display (HMD) that enables them to share the same 3D environment.
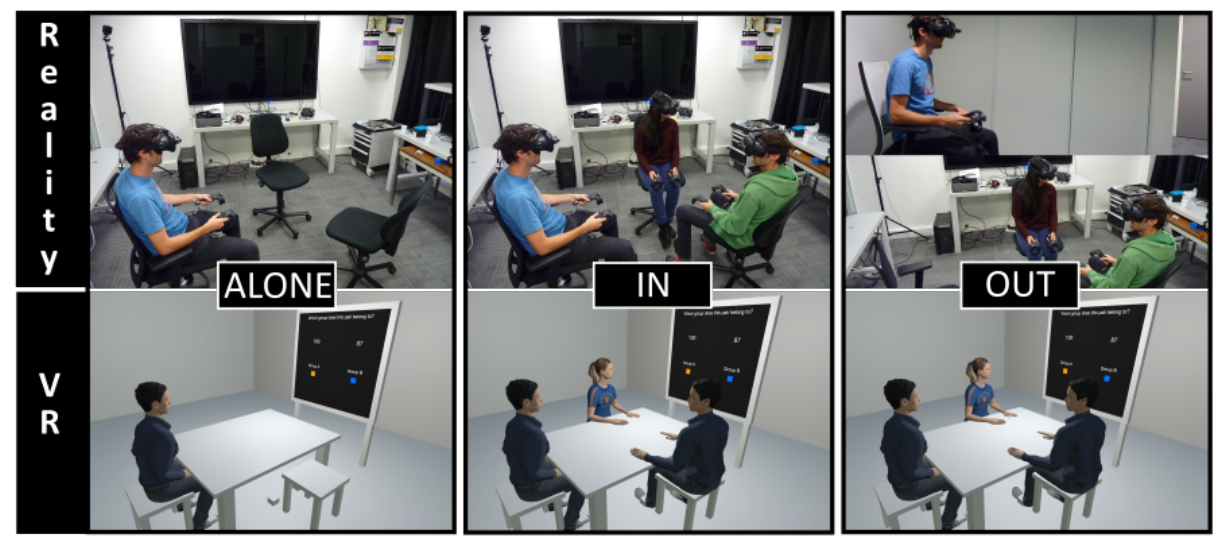

Fig. 2: Setup. User: alone (left), audience colocated (middle) or distant (right).

The evaluation concerns the user's performance (the objective measures are completion time, and type of answer), stress, cognitive workload, and perception of others (the subjective measures are based on questionnaires). Our hypothesis is that the distant audience has less impact on users than the colocated audience because the avatars of the distant audience can be perceived as agents by the user. Co-presence is lower with agents [25], reducing evaluation apprehension and self-awareness. Consistent with the experimental design and limitations of previous studies, our hypotheses are as follows:

H1 In the presence of an audience (i.e., IN, OUT), the performance (i.e., types and time of answers) of users will be diminished in comparison to the condition without an audience (i.e., ALONE).

H2 In the presence of an audience (i.e., IN, OUT), the stress and cognitive workload of users will be higher in comparison to without an audience (i.e., ALONE).

H3 Participants will feel the presence of others and their influence more when examiners are physically present in the same room (IN) rather than located in a remote room (OUT).

H4 When examiners and users are physically present in the same room (IN) rather than physically located in a remote room (OUT) the effects of the audience will be stronger (i.e., poorer performance, higher stress and cognitive workload). 


\subsection{Technical Details and Material}

Technical details. Participants were equipped with an HTC Vive and its two controllers. The VE was a virtual office with a table, a blackboard and chairs (see Fig. 2). Only three people shared the same VE. The application was built in Unity3D with the SteamVR plugin. The VE and interactions were synchronized using a software layer based on Photon Engine 4. Users and examiners were represented by human-like avatars of the same gender as themselves (see Fig. 2 ). We used a T-pose-based calibration to adjust the proportion of the avatar for each different user. The avatar animation was based on the rotation and the positions of the two controllers and the HMD using the plugin FinalIK (VRIK) 1 . The skeleton positions were inferred using inverse kinematics.

Material. Four questionnaires were used. First, the Short Stress State Questionnaire (SSSQ) [23] was used to measure task engagement, distress, and worry using a Likert scale ( $1=$ not at all; $5=$ extremely; 34 questions). Second, the Raw Task Load Index (RTLX) [9] was used to evaluate mental demand, physical demand, temporal demand, effort, performance, and frustration (6 questions). Third, the Slater-Usoh-Steed questionnaire (SUS) [42] was used to measure the feeling of presence utilizing a Likert scale $(1=$ related to low presence; $7=$ related to high presence; 6 questions). Finally, the questionnaire regarding the perception of others (QPO) was used only for assessing the conditions IN and OUT. The QPO was based on multiple co-presence questionnaires $[38,36]$. It measured two dimensions: perception of the presence of others and their perceived influence (I was in the presence of others; I forgot the others, and I was focused on the task as if I were alone; I felt observed; My performance was influenced by the presence of others in the VE), and the negative or positive impact of this perceived influence (I was embarrassed by the presence of others in the VE; The presence of others in the VE helped me perform the task; I felt embarrassed by what others might think of me). The QPO uses a Likert scale $(1=$ not at all; $7=$ extremely; 7 questions). In addition to the SSSQ, the Empatica E4 ${ }^{4}$ wristband was used to assess the cardiac activity of the user. This wristband is an unobtrusive wearable device that uses photoplethysmography signals to monitor the heart rate (HR).

\subsection{Participants}

The experiment was conducted with 57 unpaid users: 16 females and 41 males, aged from 19 to 59 years old $\left(M_{a g e}=35, s d_{a g e}=10\right)$, and with various backgrounds (i.e., students, human resources staff, engineers, managers, and assistants). Users were split into three groups: 18 participants in the ALONE condition, 20 in the IN condition, and 19 in the OUT condition. Fifteen users had never experienced VR, while 21 users had used VR less than 5 times, 14 users

\footnotetext{
${ }^{1}$ http://root-motion.com

${ }^{4}$ https://www.empatica.com/research/e4/
} 
less than 20 times, and 7 users more than 20 times. The demographic characteristics and expertise in VR of the participants were well distributed among the groups.

\subsection{Experimental Design}

The experiment involved two exercises: (1) a short tutorial and (2) the main task (see Fig. 4).

Tutorial. A description of the HTC controller was written on the blackboard: the touchpad was divided into two colored sides, left (orange) and right (blue). Then, users performed a short training task during which they were asked to click on the right or left side of the touchpad eight times (see Fig.3, left).
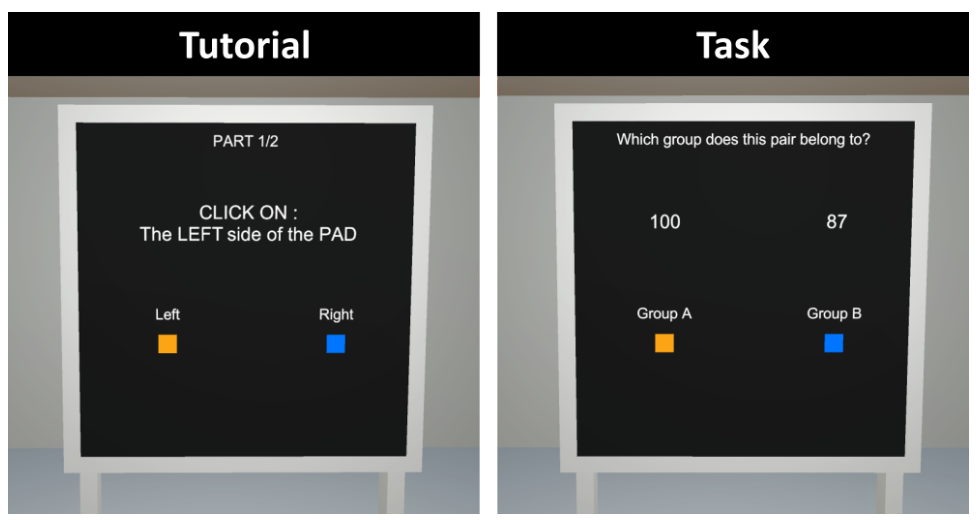

Fig. 3: Screenshots of instructions given in the tutorial and the task.

Main task. This task consisted of a categorization of numbers and was based on the work of Blascovich et al. [7] and Hoyt et al. [25]. There were two categories: numbers between 25 and 68 were included in category A, and numbers between 69 and 112 were included in category B. Participants executed several trials to discover the rules of categorization. Twenty-five trials formed a block and at least ten blocks formed the experiment. For each trial, the participant had 3 seconds to provide an answer. Specifically, in each trial, two numbers were displayed on a board in the VE, and the user was allowed 3 seconds to say whether the two numbers belonged together in category A or category B (see Fig.3, right). The possible answers were correct, categorization found $(\mathrm{OK})$; incorrect, mistake on the given categorization (NOK); or out of time, no answer given after 3 seconds (OT). The participants received audio and visual feedback: a soft beep and a green check mark for OK answers or a buzzer and a red-cross for NOK and OT answers. Category A numbers followed one normal distribution $(\mu=46.5, \sigma=8$, lower limit $=25$, upper limit $=68$ ), and category B numbers followed another 
normal distribution $(\mu=90.7, \sigma=8$, lower limit $=69$, upper limit $=112)$. The numbers were the same for each participant. After each block, the score (as a percentage) was displayed on the blackboard. To successfully complete the task, users needed to obtain $80 \%$ correct answers on two consecutive blocks. If the user did not find the categorization rule after ten blocks, the task was stopped.

\subsection{Experimental Protocol}

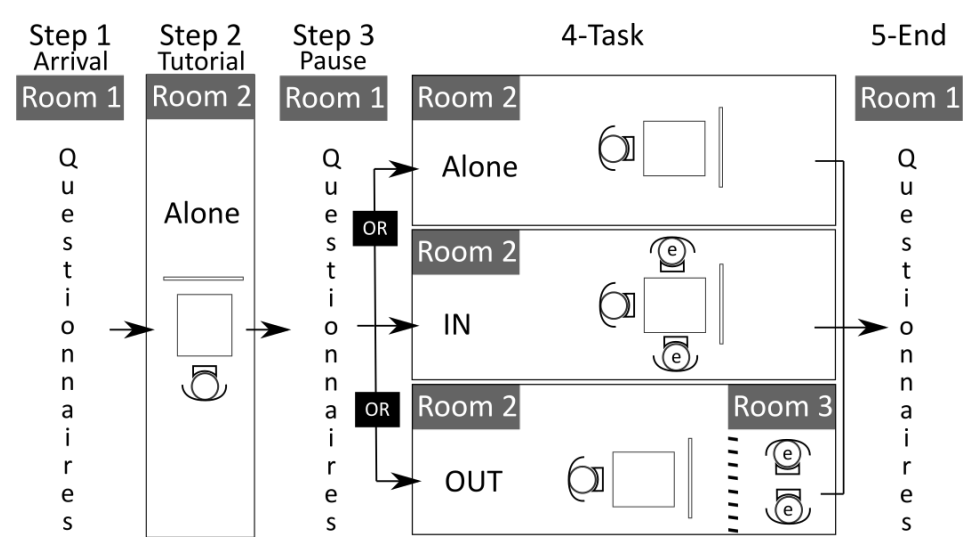

Fig. 4: Design and steps of the experiment

Step 1. All participants read and signed a consent form that briefly described the experiment and its purpose, the data recorded, the anonymity of the data, and the possibility to stop the experiment whenever the participant wished. Furthermore, each participant filled out a demographic questionnaire.

Step 2. Users were brought into a new room for the tutorial. The instructor gave information about the use of the controllers and for the calibration of the user's avatar. Then, the user was equipped with the HMD and the Empatica E4 wristband. The HR baseline was taken during the tutorial given that the user was alone, not facing others, and not performing the task. According to Fishel et al. [17], "the base-line period sets the standard against which the information of interest is compared. Setting the standard [...] referred to [...] comparing the mean output of a given standardized period of data (i.e., baseline data) with the new data of interest (e.g., experimental data)." After having equipped the user with the wristband, the instructor left the room and told the user to continue the task alone. All instructions were given on the blackboard and by a synthesized voice. The instructor then returned to notify the user that the tutorial was over.

Step 3. Users were directed to another room to fill out three questionnaires to establish their initial perceived state: the SSSQ, the RTLX, and the SUS. Then participants were redirected to the experiment room. 
Step 4. Depending on the condition, participants received different information before performing the main categorization of numbers task. The answering method was the same as in the tutorial. To elicit more social inhibition, the two examiners (i.e., one female and one male, who was also the experimenter) were introduced as an evaluative audience. During the task, examiners could only cough or move their arms, hands, and head. All noises were recorded and then played through headphones for the three conditions. Examiners were neither allowed to speak nor to answer the user. In the IN condition, participants saw and greeted the examiners before performing the task. In the OUT condition, participants never physically met the examiners. In this condition, the participants knew only that they shared the same VE as the examiners, and that the avatars embodied real people. Next, the examiners equipped themselves and invited the user to continue the experiment. Participants were also informed that their performance could not be observed by any distant person (except the audience) and that the instructor would leave the room in the OUT condition. In the IN condition, the instructor remained in the room because he was one of the two examiners. The instructions and mechanism of the task were displayed on the blackboard before beginning. The following quantitative performance data (the dependent variable) were recorded: type of answers, including number of correct $(\mathrm{OK})$, incorrect $(\mathrm{NOK})$, and out of time (OT) answers, and answering time (AT; between $0.00 \mathrm{~s}$ and $3.00 \mathrm{~s}$ ).

Step 5. Participants completed three or four questionnaires (depending on the condition) to record their final perceived state: the SSSQ, the RTLX, the SUS, and the QPO.

\subsection{Results}

As all of the trials were performed by each participant (i.e., repeated measures), linear mixed models were used [19]. To evaluate the effect of one variable using linear mixed models, two nested models were compared based on their deviance (chi-square): one without this variable (i.e., the null model), and one with this variable. We compared the effects among the three conditions (i.e., ALONE, IN, and OUT) on levels of stress, cognitive workload, and presence using KruskalWallis (not normally distributed data). Then, we performed an unpaired twosamples $t$-test (normally distributed data) to analyze differences in the levels of perceptions of others. Only the significant results of main or interaction effects $(p<.05)$ are discussed below. The main results concern the following points: performance of participants, perceptions of others, stress, cognitive workload, and presence.

Objective Performance To better evaluate variations, differences were measured between the first and the last block performed by users $(1$ block $=25$ trials, and 1 trial $=3 \mathrm{~s}$. to categorize 2 displayed numbers). It was expected that this approach would allow us to better evaluate how the behavior of users varied according to the condition in which they performed the task. Table 1 presents 
the descriptive results of the participants' performance. To conduct these analyses, we used linear mixed-effects models and generalized linear mixed-effects models to model the participants' behaviors.

Table 1: Mean and Standard Deviation of performances.

\begin{tabular}{|c|c|c|c|c|c|c|}
\hline \multirow{2}{*}{ Variable } & \multicolumn{2}{|c|}{ ALONE } & \multicolumn{2}{c|}{ IN } & \multicolumn{2}{c|}{ OUT } \\
\cline { 2 - 7 } & Mean & SD & Mean & SD & Mean & SD \\
\hline AT $(\mathrm{s}) \epsilon[0.00 ; 3.00]$ & 1.29 & .71 & 1.37 & .71 & 1.54 & .81 \\
\hline OK $(\%) \epsilon[0.00 ; 1.00]$ & .70 & .46 & .68 & .47 & .61 & .49 \\
\hline NOK $(\%) \epsilon[0.00 ; 1.00]$ & .25 & .43 & .27 & .44 & .30 & .46 \\
\hline OT $(\%) \epsilon[0.00 ; 1.00]$ & .05 & .22 & .05 & .22 & .08 & .28 \\
\hline
\end{tabular}

Answering time. For the analysis, different models are compared $\left(\mathrm{M} 0^{5}, \mathrm{M}^{6}\right.$, $\mathrm{M} 2^{7}, \mathrm{M} 3^{8}$, M4 ${ }^{9}$; see Table 2 for model comparisons for the AT variable). The analysis showed an effect of the condition over the AT (M0 vs. M1; $\chi^{2}=$ $7.88, p=.019)$ and an additive effect of the condition and trial (M1 vs. M3; $\left.\chi^{2}=1053.47, p<.001\right)$. The answering time varied from one trial to an other (see Fig. 5). Comparisons between conditions (post-hoc tests) showed a significant difference between ALONE and OUT $(z=-2.76, p=.015)$, partially supporting H1. There was no significant interaction effect (M3 vs. M4; $\left.\chi^{2}=3.23, p=.198\right)$. In other words, the AT was diminished when the audience was distant as opposed to when no audience was present. The comparisons between OUT and IN and between IN and ALONE were not significant; H1 and $\mathbf{H} \mathbf{4}$ are not supported. The AT was not significantly diminished when the audience was colocated as opposed to when no audience was present, and similar results were obtained for the distant audience compared to the colocated audience condition.

Type of answers. We conducted the analyses on the number of OK, NOK, and OT answers given by participants between models. The values registered for each answer were as follows: for OK, 0 (i.e., not correct) or 1 (i.e., correct); for NOK, 1 (i.e., incorrect) or 0 (i.e., not incorrect); and for OT, 1 (i.e., out of time)

${ }^{5} \mathrm{M} 0$ is the null model only with the random effect of the participant: $M 0=A T \sim(1 \mid$ Participant $)$.

${ }^{6} \mathrm{M} 1$ is the model with the random effect of the participant and the effect of the condition: $M 1=A T \sim$ Condi $+(1 \mid$ Participant $)$.

${ }^{7} \mathrm{M} 2$ is the model with the random effect of the participant and the effect of the trial: $M 2=A T \sim$ Trial $+(1 \mid$ Participant $)$.

${ }^{8} \mathrm{M} 3$ is the model with the random effect of the participant plus the effect of the condition and the effect of the trial: $M 3=A T \sim$ Condi + Trial $+(1 \mid$ Participant $)$.

${ }^{9} \mathrm{M} 4$ is the model with the random effect of the participant plus the effect of the condition and the effect of the trial and their interaction: $M 4=A T \sim$ Condi $*$ Trial $+(1 \mid$ Participant $)$. 
Table 2: Comparisons of models - answering time.

\begin{tabular}{|c|c|c|c|c|}
\hline Compared Models & DV & Evaluated Effect & $\chi^{2}$ & p-value \\
\hline M0 vs. M1 & AT & CONDI & 7.88 & .019 \\
\hline M1 vs. M3 & AT & CONDI+TRIAL & 1053.47 & $<.001$ \\
\hline M3 vs. M4 & AT & CONDI $\times$ TRIAL & 3.23 & .198 \\
\hline
\end{tabular}

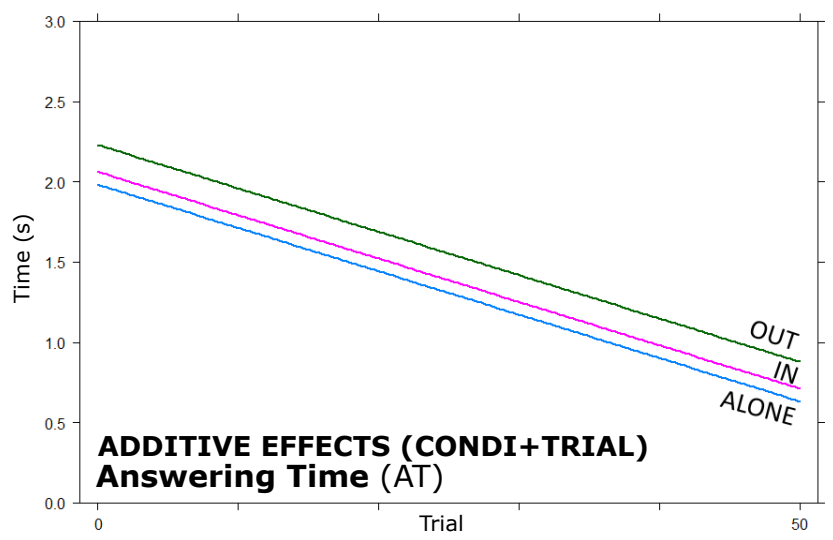

Fig. 5: Additive effects of Trial and Condition on answering time

or 0 (i.e., in time). We used mixed-effects logistic regression with random effects. Table 3 presents the results of comparisons of models for the variables OK, NOK, and OT. The effect of the trial was significant over the number of OK, NOK, OT answers (M0 vs. M2 models; $\chi_{o k}^{2}=351.45, p_{o k}<.001 ; \chi_{n o k}^{2}=146.81, p_{n o k}<$ $\left..001 ; \chi_{o t}^{2}=183.49, p_{o t}<.001\right)$. The interaction effects between the condition and the trial were also significant (M3 vs. M4 models; $\chi_{o k}^{2}=15.25, p_{o k}<.001$; $\left.\chi_{n o k}^{2}=11.09, p_{n o k}=.004 ; \chi_{o t}^{2}=18.33, p_{o t}<.001\right)$.

The number of OK answers grew more quickly in the ALONE condition than in the IN and OUT conditions (see Fig. 6). The number of NOK and OT answers decreased more quickly in the ALONE condition than in the IN and OUT conditions. This interaction between the conditions and trials (CONDI*TRIAL) in these three variables supports $\mathbf{H 1}$, but not $\mathbf{H} 4$. In other words, the evolution of the type of answers over trials was significantly different in relation to the location of the audience. Specifically, the number of OK answers increased more slowly over the trials in the presence of the audience (i.e., IN or OUT) as opposed to without an audience present (i.e., ALONE), but the evolution occurred more quickly in the presence of the colocated audience compared to the distant audience condition. Furthermore, the number of NOK and OT answers decreased more slowly over trials in the presence of the audience (i.e., IN or OUT) as opposed to trials without the audience present (i.e., ALONE), but 
Table 3: Comparisons of models - three types of answer.

\begin{tabular}{|c|c|c|c|c|}
\hline Compared Models & DV & Evaluated Effect & $\chi^{2}$ & p-value \\
\hline M0 vs. M2 & OK & TRIAL & 351.45 & $<.001$ \\
\hline M2 vs. M3 & OK & CONDI+TRIAL & 2.46 & .292 \\
\hline M3 vs. M4 & OK & CONDI $\times$ TRIAL & 15.25 & $<.001$ \\
\hline \hline M0 vs. M2 & NOK & TRIAL & 146.81 & $<.001$ \\
\hline M2 vs. M3 & NOK & CONDI+TRIAL & 1.03 & .596 \\
\hline M3 vs. M4 & NOK & CONDI $\times$ TRIAL & 11.09 & .004 \\
\hline \hline M0 vs. M2 & OT & TRIAL & 183.49 & $<.001$ \\
\hline M2 vs. M3 & OT & CONDI+TRIAL & 7.50 & .024 \\
\hline M3 vs. M4 & OT & CONDI $\times$ TRIAL & 18.33 & $<.001$ \\
\hline
\end{tabular}

the evolution occurred more quickly in the presence of the colocated audience compared to the distant audience condition.

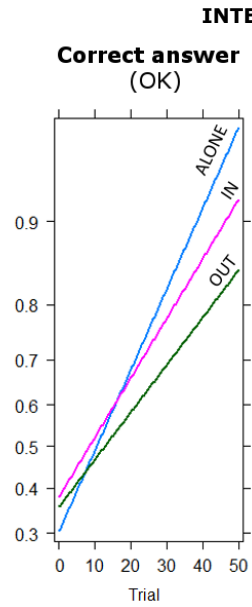

NTERACTION EFFECTS (CONDI*TRIAL)
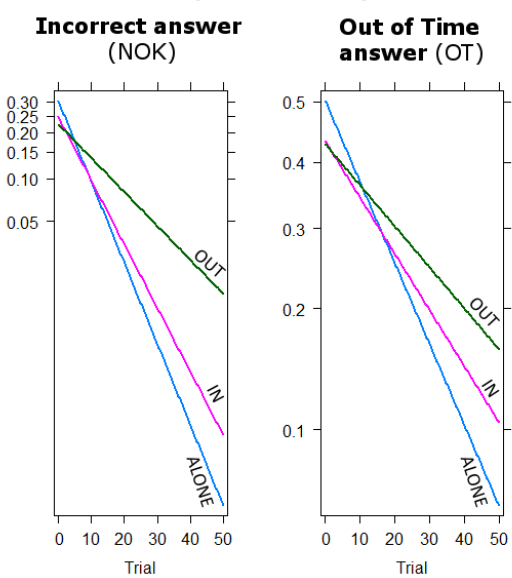

Fig. 6: Interaction effects of Trial and Condition on type of answers

Subjective Feelings and Perceptions Perception of others. Using the QPO, the $t$-test showed a main effect on the Perception of Others and their Influence $(t(31.33)=2.43, p=.021)$ (see Fig. 7$)$. Users perceived the examiners more significantly and felt more influenced by them when the examiners were physically colocated $\left(M_{\text {in }}=4.99, s d_{i n}=0.83\right)$ than when they were distant $\left(M_{\text {out }}=4.09\right.$, 
$\left.s d_{\text {out }}=1.40\right)$. No other significant difference was found. H3 is supported by these results.
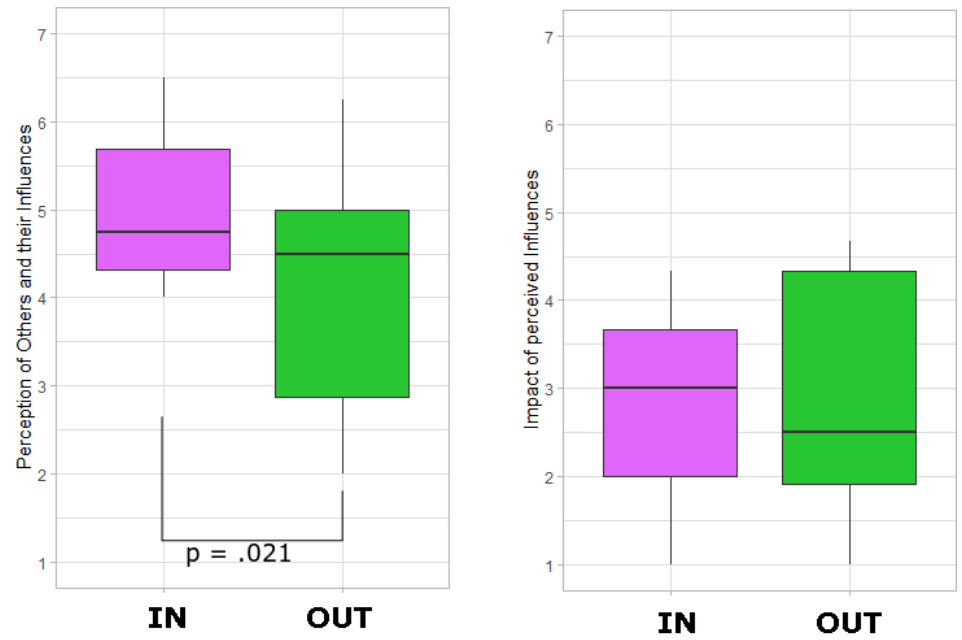

Fig. 7: Boxplots, per condition, of scores from the QPO

Stress. The Kruskal-Wallis tests did not show significant differences regarding all dimensions of the SSSQ: Engagement $\left(M_{\text {alone }}=3.75, s d_{\text {alone }}=1.13\right.$; $\left.M_{\text {in }}=3.46, s d_{\text {in }}=1.11 ; M_{\text {out }}=3.54, s d_{\text {out }}=1.30\right)$, Worry $\left(M_{\text {alone }}=1.78\right.$, $\left.s d_{\text {alone }}=1.02 ; M_{\text {in }}=1.99, s d_{\text {in }}=1.17 ; M_{\text {out }}=2.12, s d_{\text {out }}=1.22\right)$, and Distress $\left(M_{\text {alone }}=1.83, s d_{\text {alone }}=1.07 ; M_{\text {in }}=1.69, s d_{\text {in }}=1.03 ; M_{\text {out }}=1.86\right.$, $\left.s d_{\text {out }}=1.10\right)$. $\mathbf{H} \mathbf{2}$ and $\mathbf{H} 4$ are not supported by these results. In other words, participants' stress levels were not significantly affected by audience location.

Additionally, we used HR measurements in beats per minute (bpm) to explore variability during the task performance. We extracted the mean of the HR from the baseline and we extracted the mean of the HR from each block. Due to the inter-individual variability, we subtracted the mean of the baseline from the mean of the blocks ${ }^{10}$. Table 4 reports the descriptive results (means, standard deviations, and boxplots) of these physiological measurements. No significant difference was found (Kruskal-Wallis tests) regarding DHRi between conditions. These results do not support H2 and H4. In other words, participants' HR measurements were not significantly different in relation to the location of the audience.

Cognitive workload. The RTLX questionnaire had six dimensions: Mental Demand, Physical Demand, Temporal Demand, Effort, Performance, and Frus-

${ }^{10}$ Equation to obtain the value used of the HR for analyses: $D H R_{\text {block }_{i}}=H R_{\text {block }_{i}}-$ $H R_{\text {baseline }}$. 
Table 4: Means and Standard Deviations for recorded data of HR.

\begin{tabular}{|c|c|c|c|c|c|c|}
\hline \multirow{2}{*}{ Variable } & \multicolumn{2}{|c|}{ Cosi } & \multicolumn{2}{c|}{ Dist } & \multicolumn{2}{c|}{ Alone } \\
\cline { 2 - 7 } & Mean & SD & Mean & SD & Mean & SD \\
\hline$H R_{\text {baseline }}(\mathrm{bpm})$ & $\mathbf{8 1 . 3}$ & $\mathbf{1 6 . 1 3}$ & $\mathbf{7 6 . 1 5}$ & $\mathbf{1 1 . 2 9}$ & $\mathbf{7 3 . 0 1}$ & $\mathbf{8 . 7 1}$ \\
\hline$D H R_{\text {block }_{1}}(\mathrm{bpm})$ & $\mathbf{7 . 5 5}$ & $\mathbf{6 . 9 2}$ & $\mathbf{6 . 2 9}$ & $\mathbf{1 0 . 4 3}$ & $\mathbf{4 . 0 5}$ & $\mathbf{5 . 0 1}$ \\
\hline$D H R_{\text {block }_{2}}(\mathrm{bpm})$ & $\mathbf{3 . 9 8}$ & $\mathbf{5 . 7 8}$ & $\mathbf{3 . 9 3}$ & $\mathbf{9 . 0 1}$ & $\mathbf{2 . 4 7}$ & $\mathbf{4 . 8 5}$ \\
\hline
\end{tabular}

tration. Only the means of Physical Demand were low $\left(M_{A L O N E}=16.32\right.$, $s d_{A L O N E}=15.80, M_{I N}=15.28, s d_{I N}=16.04$, and $M_{O U T}=11.00, s d_{O U T}=$ $10.95)$, while the means of the other dimensions varied around the middle score of $50.00(M \epsilon[42.00 ; 62.00])$. The Kruskal-Wallis tests did not find significant differences regarding all dimensions of the RTLX. H2 and $\mathbf{H 4}$ are not supported by these results. In other words, participants' workload was not significantly affected by audience location.

Presence. The SUS questionnaire had two scores: Mean and Count (i.e., number of scores equal to or higher than 6 ). The one-way ANOVA test did not find significant differences regarding all scores of the SUS. Users felt a moderate presence $\left(M_{A L O N E}=3.5, s d_{A L O N E}=2.05, M_{I N}=4.10, s d_{I N}=1.88, M_{O U T}=4.17\right.$, $\left.s d_{O U T}=1.79\right)$.

Gender effect. The gender effect was evaluated for objective performance and for subjective feelings and perceptions. No significant effect was found outside on the Engagement (dimension of SSSQ). Indeed, women were significantly less engaged than men $\left(M_{\text {women }}=3.27, s d_{\text {women }}=0.75 ; M_{\text {men }}=3.70, s d_{\text {men }}=\right.$ $\left.0.64 ; \chi^{2}(2)=4.05, p<.044\right)$.

\section{Discussion}

The results show that the presence of the examiners influenced the participant's natural performance improvement (i.e., answering time and type of answers) when performing a repeated task.

First, objective measures show a significant improvement of correct answers within the time frame as well as a reduction of false and out of time answers (i.e. "OK", "NOK", and "OT"). This can be explained by a natural improvement due to repetitions during the task. But the improvement is not the same between conditions. The interaction effects between the audience and the trials showed that the evolution within the time frame, whether positive or negative (depending on the objective measure), was slower when an audience was present compared to absent. The manifestation of social inhibition among users due to the presence of an audience seemed to result in a decrease of the natural performance evolution during this specific task. Our results are consistent with 
previous social inhibition studies [25]. Thus, $\mathbf{H 1}$ is partially supported because answering time results show no clear distinction between the presence or absence of the audience. The only significant difference is between the distant and the colocated audiences.

Second, social inhibition is not perceptible in the participants' cognitive workload and stress. The results of the questionnaires did not demonstrate any differences, despite the anxiety about an evaluative audience that could have been induced by the presence of the examiners. Thus, $\mathbf{H 2}$ is not supported. This result could be due to the variability of mathematical skill among the participants [27]. Indeed, the perceptual abilities and resources used to perform the task may vary between different participants. Unfortunately, mathematical aptitude was not evaluated in the current study.

Third, results comparing participants' subjective perception of others between colocated (i.e., IN) and distant (i.e., OUT) audiences show significant differences. Participants seemed to perceive the examiners more significantly and appeared to feel more influenced by them when the examiners were physically colocated than when they were distant. Thus, H3 is supported. However, participants did not find significant differences between conditions that resulted in a negative influence. Moreover, the overall score of the negative influence of the examiners was probably not high because the examiners were passive during the experiment and did not cause any additional stress.

Finally, users' performance refutes $\mathbf{H} 4$. The statistical analysis demonstrated significant differences between conditions (i.e., IN, and OUT). Indeed, previous interaction effects (i.e., audience over trial) also show that the positive or negative evolution of the performance occurred even more slowly when the users and the audience were distant. Therefore, a stronger social inhibition seems to occur in distant users compared to users who are next to one another. This finding is most likely the explanation for the slower natural performance improvement (i.e., type of answers) of the participants. The results seem to indicate that the reason users negatively experience their performance (when all participants and examiners are in the same real room) is not related to the extent to which they perceive or feel the presence of others. To sum up, the performance of the participants seems to be affected by both the presence of an audience and the type of the audience (i.e., IN, OUT). Furthermore, the significance of the participants' perceptions of others seems to also depend on the type of the audience.

\section{Conclusion}

Our goal was to investigate differences in social inhibition. An experimental study was conducted to induce this social effect in two different conditions. The user and the audience shared the same virtual environment, with the difference in the conditions consisting in the real audience's location, which was either a different or the same room as the user. Performance and subjective results were obtained during an unknown and challenging task. The results of the two 
conditions were compared with a control condition (i.e., the user alone). Two major outcomes have been found and no gender effect hase been detected.

First, the presence of observers causes a difference in users' reactions compared to when users are alone in the VE. Indeed, the natural performance improvement occurred more slowly when the audience was colocated than when no audience was present, and even more slowly with a distant audience than with a colocated audience. A slower improvement with a distant audience could be due to a weaker perception of others compared to a colocated audience.

Second, and more generally, social inhibition seems to occur when the users in VR are in the presence of an audience. Moreover, in a remote room, the physically distant audience seems to affect the users' social inhibition even more strongly. A learning or training process using repetition could therefore be influenced by the presence of an audience. The designers of such applications should pay attention to this aspect of the setup before building the application.

As the user's personality could impact their perceptions, it could be interesting to explore the variation of social inhibition among different personality traits and through the same audience types. The type of the task could also be investigated by analyzing, for example, the variation of social inhibition in fire drills, training for maintenance, and evaluation of a service. The mere presence of an audience in reality, without its presence in VR, could also be investigated corresponding to a demonstration in an exhibition scenario.

Acknowledgement This study was carried out within $\mathrm{b}<>$ com, an institute of research and technology dedicated to digital technologies. It received support from the Future Investments program of the French National Research Agency (grant no. ANR-07-A0-AIRT).

\section{References}

1. Argelaguet Sanz, F., Multon, F., Lécuyer, A.: A methodology for introducing competitive anxiety and pressure in vr sports training. Frontiers in Robotics and AI 2, 10 (2015)

2. Aymerich-Franch, L., Bailenson, J.: The use of doppelgangers in virtual reality to treat public speaking anxiety: a gender comparison. In: Proceedings of the International Society for Presence Research Annual Conference. pp. 173-186. Citeseer (2014)

3. Bailenson, J.N., Beall, A.C., Blascovich, J.: Gaze and task performance in shared virtual environments. The Journal of Visualization and Computer Animation 13(5), 313-320 (2002)

4. Bailenson, J.N., Segovia, K.Y.: Virtual Doppelgangers: Psychological Effects of Avatars Who Ignore Their Owners, pp. 175-186. Springer London, London (2010)

5. Bailenson, J.N., Yee, N., Blascovich, J., Beall, A.C., Lundblad, N., Jin, M.: The use of immersive virtual reality in the learning sciences: Digital transformations of teachers, students, and social context. Journal of the Learning Sciences 17(1), 102-141 (2008) 
6. Blascovich, J., Loomis, J., Beall, A.C., Swinth, K.R., Hoyt, C.L., Bailenson, J.N.: Target article: Immersive virtual environment technology as a methodological tool for social psychology. Psychological Inquiry 13(2), 103-124 (2002)

7. Blascovich, J., Mendes, W.B., Hunter, S.B., Salomon, K.: Social "facilitation" as challenge and threat. Journal of personality and social psychology 77(1), 68 (1999)

8. Bond, C.F., Titus, L.J.: Social facilitation: A meta-analysis of 241 studies. Psychological bulletin 94(2), 265 (1983)

9. Byers, J.C.: Traditional and raw task load index (tlx) correlations: Are paired comparisons necessary? Advances in Industrial Ergonomics and Safety l: Taylor and Francis (1989)

10. Campos-Castillo, C.: Copresence in virtual environments. Sociology Compass 6(5), 425-433 (2012). https://doi.org/10.1111/j.1751-9020.2012.00467.x

11. Chartrand, T.L., Bargh, J.A.: The chameleon effect: the perception-behavior link and social interaction. Journal of personality and social psychology $\mathbf{7 6}(6), 893$ (1999)

12. Claypoole, V.L., Dewar, A.R., Fraulini, N.W., Szalma, J.L.: Effects of social facilitation on perceived workload, subjective stress, and vigilance-related anxiety. Proceedings of the Human Factors and Ergonomics Society Annual Meeting 60(1), 1169-1173 (2016)

13. Cottrell, N.B., Wack, D.L., Sekerak, G.J., Rittle, R.H.: Social facilitation of dominant responses by the presence of an audience and the mere presence of others. Journal of personality and social psychology 9(3), 245 (1968)

14. Dijksterhuis, A., Bargh, J.A.: The perception-behavior expressway: Automatic effects of social perception on social behavior. In: Advances in Experimental Social Psychology, vol. 33, pp. 1 - 40. Academic Press (2001)

15. Durlach, N., Slater, M.: Presence in shared virtual environments and virtual togetherness. Presence: Teleoperators and Virtual Environments 9(2), 214-217 (2000)

16. Emmerich, K., Masuch, M.: Watch me play: Does social facilitation apply to digital games? In: Proceedings of the 2018 CHI Conference on Human Factors in Computing Systems. pp. 100:1-100:12. CHI '18 (2018)

17. Fishel, S.R., Muth, E.R., Hoover, A.W.: Establishing appropriate physiological baseline procedures for real-time physiological measurement. Journal of Cognitive Engineering and Decision Making 1(3), 286-308 (2007). https://doi.org/10.1518/155534307X255636

18. Garau, M., Slater, M., Pertaub, D.P., Razzaque, S.: The responses of people to virtual humans in an immersive virtual environment. In: Presence: Teleoperators and Virtual Environments. vol. 14, pp. 104-116 (2005)

19. Gueorguieva, R., Krystal, J.H.: Move over anova: Progress in analyzing repeatedmeasures data and its reflection in papers published in the archives of general psychiatry. Archives of General Psychiatry 61(3), 310-317 (Mar 2004)

20. Guerin, B., Innes, J.M.: Social facilitation and social monitoring: A new look at zajonc's mere presence hypothesis. British Journal of Social Psychology 21(1), 7-18 (1982)

21. Guerin, B.: Mere presence effects in humans: A review. Journal of experimental social psychology 22(1), 38-77 (1986)

22. Hartanto, D., Kampmann, I.L., Morina, N., Emmelkamp, P.G.M., Neerincx, M.A., Brinkman, W.P.: Controlling social stress in virtual reality environments. PLOS ONE 9(3), 1-17 (03 2014)

23. Helton, W.S., Näswall, K.: Short stress state questionnaire. European Journal of Psychological Assessment 31(1), 20-30 (jun 2015) 
24. Henchy, T., Glass, D.C.: Evaluation apprehension and the social facilitation of dominant and subordinate responses. Journal of personality and social psychology 10(4), $446(1968)$

25. Hoyt, C.L., Blascovich, J., Swinth, K.R.: Social inhibition in immersive virtual environments. Presence: Teleoperators and Virtual Environments 12(2), 183-195 (2003)

26. Innes, J.M., Young, R.F.: The effect of presence of an audience, evaluation apprehension and objective self-awareness on learning. Journal of Experimental Social Psychology (1975)

27. Jamieson, J.P., Peters, B.J., Greenwood, E.J., Altose, A.J.: Reappraising stress arousal improves performance and reduces evaluation anxiety in classroom exam situations. Social Psychological and Personality Science 7(6), 579-587 (2016)

28. Knowles, E.S.: Social physics and the effects of others: Tests of the effects of audience size and distance on social judgments and behavior. Journal of Personality and Social Psychology 45(6), 1263 (1983)

29. Kushnir, T.: Stress and social facilitation: the effects of the presence of an instructor on student nurses' behaviour. Journal of Advanced Nursing 11(1), 13-19 (1986)

30. Lemoine, J.E., Roland-Lévy, C.: The effect of the presence of an audience on risktaking while gambling: the social shield. Social Influence 12(2-3), 101-114 (2017)

31. Sanchez-Vives, M.V., Slater, M.: From presence to consciousness through virtual reality. Nature Reviews Neuroscience 6(4), 332 (2005)

32. Schroeder, R.: Being there together and the future of connected presence. Presence: Teleoperators and Virtual Environments 15(4), 438-454 (2006)

33. Shriram, K., Oh, S.Y., Bailenson, J.: 22 virtual reality and prosocial behavior. Social signal processing p. 304 (2017)

34. Skarratt, P.A., Cole, G.G., Kingstone, A.: Social inhibition of return. Acta Psychologica 134(1), 48 - 54 (2010)

35. Slater, M., Antley, A., Davison, A., Swapp, D., Guger, C., Barker, C., Pistrang, N., Sanchez-Vives, M.V.: A virtual reprise of the stanley milgram obedience experiments. PLOS ONE 1(1), 1-10 (12 2006). https://doi.org/10.1371/journal.pone.0000039

36. Slater, M., Sadagic, A., Usoh, M., Schroeder, R.: Small-group behavior in a virtual and real environment: A comparative study. Presence: Teleoperators and Virtual Environments 9(1), 37-51 (2000)

37. Smeesters, D., Wheeler, S.C., Kay, A.C.: Indirect prime-to-behavior effects: The role of perceptions of the self, others, and situations in connecting primed constructs to social behavior. In: Advances in Experimental Social Psychology, vol. 42, pp. 259 - 317. Academic Press (2010)

38. Steed, A., Slater, M., Sadagic, A., Bullock, A., Tromp, J.: Leadership and collaboration in shared virtual environments. In: Proceedings IEEE Virtual Reality (Cat. No. 99CB36316). pp. 112-115 (March 1999)

39. Stotland, E., Zander, A.: Effects of public and private failure on self-evaluation. The Journal of Abnormal and Social Psychology 56(2), 223-229 (1958)

40. Swinth, K.R., Blascovich, J.: Perceiving and responding to others: Human-human and human-computer social interaction in collaborative virtual environments. In: Proceedings of the 5th Annual International Workshop on PRESENCE. vol. 392 (2002)

41. Terrier, R., Martin, N., Lacoche, J., Gouranton, V., Arnaldi, B.: Am i better in vr with a real audience? In: Gavrilova, M., Chang, J., Thalmann, N.M., Hitzer, E., Ishikawa, H. (eds.) Advances in Computer Graphics. pp. 28-39. Springer International Publishing, Cham (2019) 
42. Usoh, M., Catena, E., Arman, S., Slater, M.: Using presence questionnaires in reality. Presence: Teleoperators \& Virtual Environments 9(5), 497-503 (2000)

43. Velho, L., Lucio, D., Carvalho, L.: Situated participatory virtual reality. In: In Proceedings of XVI Simposio Brasileiro de Jogos e Entretenimento Digital (2017)

44. Wienrich, C., Gross, R., Kretschmer, F., Müller-Plath, G.: Developing and proving a framework for reaction time experiments in vr to objectively measure social interaction with virtual agents. In: 2018 IEEE Conference on Virtual Reality and 3D User Interfaces (VR). pp. 191-198 (March 2018)

45. Wolf, L.K., Bazargani, N., Kilford, E.J., Dumontheil, I., Blakemore, S.J.: The audience effect in adolescence depends on who's looking over your shoulder. Journal of Adolescence 43, 5- 14 (2015)

46. $\mathrm{Yu}, \mathrm{R} . \mathrm{f} ., \mathrm{Wu}, \mathrm{X} .:$ Working alone or in the presence of others: exploring social facilitation in baggage x-ray security screening tasks. Ergonomics 58(6), 857-865 (2015)

47. Zajonc, R.B.: Social facilitation. Science 149(3681), 269-274 (1965) 